Société d'histoire de la révolution de 1848 et des révolutions du XIXe siècle

\title{
Colloque: Hugo politique. Programme
}

\section{(2) OpenEdition \\ Journals}

Édition électronique

URL : http://journals.openedition.org/rh19/348

DOI : $10.4000 /$ rh 19.348

ISSN : $1777-5329$

\section{Éditeur}

La Société de 1848

\section{Édition imprimée}

Date de publication : 1 décembre 2001

Pagination : $317-320$

ISSN : 1265-1354

Référence électronique

" Colloque: Hugo politique. Programme », Revue d'histoire du XIXe siècle [En ligne], 23 | 2001, mis en ligne le 15 octobre 2002, consulté le 15 septembre 2020. URL : http://journals.openedition.org/ rh19/348

Ce document a été généré automatiquement le 15 septembre 2020.

Tous droits réservés 


\title{
Colloque: Hugo politique. Programme
}

\author{
Université de Franche-Comté \\ BICENTENAIRE DE LA NAISSANCE DE VICTOR HUGO \\ COLLOQUE INTERNATIONAL
}

Besançon

11, 12, 13 décembre 2002

Petit Kursaal

Avec le soutien de la ville de Besançon, du Conseil régional de Franche-Comté, de la DRAC de Franche-Comté et de l'Université de Franche-Comté

"Victor Hugo a célébré la suprématie sous toutes les formes possibles : d'abord la suprématie admise : tradition, monarchie, légitimité, religion révélée; ensuite l'action autocratique du génie dans la figure de Napoléon, puis l'image idéalisée du Peuple, la suprématie morale du proscrit intransigeant, du banni sublime, enfin toutes les forces de la Nature et encore une fois la simplicité de l'enfant, de l'animal".

Hugo von Hofmannsthal, Victor Hugo, 1900

Victor Hugo a, de manière consciente, inscrit sa vie dans un cadre séculaire, le XIX ${ }^{\mathrm{e}}$ siècle. Au moment où s'ouvre un nouveau siècle, il est important de rappeler la mémoire d'un Hugo politique dont la pensée entretient un rapport étroit avec la question sociale, l'émergence de la démocratie, l'invention de l'Europe, l'idée de justice, etc. La mort de Hugo a été ressentie et célébrée, en France et bien au delà, comme un événement considérable, sorte d'apothéose de la République et de la démocratie triomphantes : au risque de réduire le parcours de l'homme à sa dernière étape, ou d'opérer un tri dans sa production politique. Au cœur de ce colloque figure donc la dimension politique de Hugo. Ses œuvres de jeunesse comme celles de la maturité et de la vieillesse interrogent et influencent l'évolution politique de la France, mais aussi de l'Europe, dont il prévoit l'avènement des États-Unis. Dans son journal comme dans ses romans, dans ses poèmes ou ses tragédies comme dans ses discours politiques, Hugo mobilise toutes les formes de l'écriture au service des causes qu'il défend. S'il envisage la société de son temps au travers de ses injustices et de ses inégalités, il entrevoit celle de l'avenir au travers des notions de droit et de justice. 
S'agit-il, en définitive, d'une pensée politique de même nature que celle de ses contemporains qui imaginent un monde à reconstruire --comme les utopistes-- ou qui sont des professionnels de la politique ? Cela renvoie à un questionnement sur la cohérence de la pensée politique de l'écrivain et, au delà, sur la cohérence de l'attitude de Hugo vis-à-vis du politique et de la politique.

\title{
Comité scientifique
}

Maurice Agulhon, Victor Brombert, Jean Gaudon, Tony James, Bernard Leuilliot, Mona Ozouf, Michelle Perrot, Guy Rosa, Jacques Seebacher, Agnès Spiquel, Anne Ubersfeld.

\section{Comité d'organisation}

Gaston Bordet, Jean-Claude Caron, Pierre Laforgue, François Marcot, Annie Stora-

Lamarre.

\section{Secrétariat du colloque}

Mme Marie-Claude Charles,

Faculté des lettres, secrétariat du colloque Hugo politique, laboratoire des sciences historiques,

30, rue Mégevand 25030 Besançon Cedex.

Téléphone/Fax : 0381665433.

E-mail : marie-claude.charles@univ-fcomte.fr

Programme

\section{Mercredi 11 décembre 2002}

\begin{tabular}{|l|l|}
\hline $14 \mathrm{~h}$ & Accueil des participants \\
\hline & Allocution du Maire de Besançon \\
\hline & Ouverture du colloque par Eliane VERGNOLLE \\
\hline & Présentation générale : Jean-Claude CARON et Pierre LAFORGUE \\
\hline
\end{tabular}

$1^{\text {ère }}$ session : ACTES ET PAROLES

Président de séance : Maurice AGULHON

\begin{tabular}{|l|l|}
\hline $14 \mathrm{~h} 30$ & Bernard DEGOUT, "Le pardon de Didier. Victor Hugo et la Restauration" \\
\hline $15 \mathrm{~h}$ & $\begin{array}{l}\text { Marieke STEIN, "'Je resterai indépendant, dussé-je rester isolé". Victor Hugo et les partis } \\
\text { politiques sous la Seconde République" }\end{array}$ \\
\hline $15 \mathrm{~h} 30$ & Bernard LEUILLIOT, "Victor Hugo, "socialiste" de la veille et des lendemains" \\
\hline $16 \mathrm{~h}$ & Pause \\
\hline $16 \mathrm{~h} 15$ & Guy ROSA, "Politique de Hugo exilé" \\
\hline $16 \mathrm{~h} 45$ & Agnès SPIQUEL, "Hugo et Gambetta (1871-1882)" \\
\hline $17 \mathrm{~h} 15$ & Suzanna BARROWS, "Victor Hugo et le Coup du Seize mai" \\
\hline
\end{tabular}

Discussion

Jeudi 12 décembre 2002

\author{
$2^{\mathrm{e}}$ session : DROIT ET JUSTICE CHEZ HUGO \\ Présidente de séance : Michelle PERROT
}




\begin{tabular}{|l|l|}
\hline $9 \mathrm{~h}$ & Jean GAUDON, "Politique et vandalismes" \\
\hline $9 \mathrm{~h} 30$ & $\begin{array}{l}\text { Jean-Claude CARON, "Violences vues, violences vécues. Hugo face à la violence politico- } \\
\text { sociale" }\end{array}$ \\
\hline $10 \mathrm{~h}$ & $\begin{array}{l}\text { Jean-Claude FIZAINE, "Légitimité politique et responsabilité individuelle : pratique et } \\
\text { théorie, 1850-1860" }\end{array}$ \\
\hline $10 \mathrm{~h} 30$ & Pause \\
\hline $10 \mathrm{~h} 45$ & Odile KRAKOVITCH, "Hugo et la liberté théâtrale" \\
\hline $11 \mathrm{~h} 15$ & Dominique KALIFA, "Javert enquêteur" \\
\hline $11 \mathrm{~h} 45$ & Annie STORA-LAMARRE, "La puissance du droit dans Les Misérables" \\
\hline
\end{tabular}

Discussion

\section{$3^{\mathrm{e}}$ session : FONDEMENTS INTELLECTUELS DE LA PENSEE HUGOLIENNE}

Président de séance : Guy ROSA

\begin{tabular}{|l|l|}
\hline $14 \mathrm{~h}$ & Anne UBERSFELD, "Hugo et la parole du peuple" \\
\hline $14 \mathrm{~h} 30$ & Mona OZOUF, "Le mot monarchie et la chose république. 1830-1848" \\
\hline $15 \mathrm{~h}$ & Thomas BOUCHET, "Figures libres. L'orateur Hugo, jusqu'à l'exil" \\
\hline $15 \mathrm{~h} 30$ & Pause \\
\hline $15 \mathrm{~h} 45$ & Pierre LAFORGUE, "Philosophie et politique (1860-1865)" \\
\hline $16 \mathrm{~h} 15$ & Gaston BORDET, "Victor Hugo et la papauté. La virulence laïque d'un écrivain déçu" \\
\hline
\end{tabular}

Discussion \begin{tabular}{|l|l}
\hline 17 h30 & $\begin{array}{l}\text { Conférence de Jacques SEEBACHER : "Libéralisme et socialisme : la tentation } \\
\text { communaliste chez Hugo et Girardin" }\end{array}$
\end{tabular}

\begin{tabular}{|l|l|}
\hline $19 \mathrm{~h}$ & Réception à la Mairie \\
\hline
\end{tabular}

\section{Vendredi 13 décembre 2002}

$4^{\mathrm{e}}$ session : UNIVERSALISME DE HUGO

Président de séance : Jacques SEEBACHER

\begin{tabular}{|l|l|}
\hline $9 \mathrm{~h}$ & Franck LAURENT, "La politique allemande de Victor Hugo" \\
\hline $9 \mathrm{~h} 30$ & Jonathan BEECHER, "Victor Hugo et Alexandre Herzen : deux frères d'exil" \\
\hline $10 \mathrm{~h}$ & $\begin{array}{l}\text { Gilles PECOUT, "Visages de Victor Hugo dans l'Italie libérale, des lendemains de l'Unité à } \\
\text { la Première Guerre mondiale" }\end{array}$ \\
\hline $10 \mathrm{~h} 30$ & Pause \\
\hline $10 \mathrm{~h} 45$ & Robert DAMIEN, "Hugo et le problème politique de la bibliothèque publique" \\
\hline
\end{tabular}


\begin{tabular}{|l|l}
\hline $11 \mathrm{~h} 15$ & Michel VERNUS, "Le livre dans l'œuvre de Victor Hugo"
\end{tabular}

Discussion

$5^{\mathrm{e}}$ session : MEMOIRE ET IMAGINAIRE DE HUGO

Président de séance : Gaston BORDET

\begin{tabular}{|l|l|}
\hline $14 \mathrm{~h}$ & Dolf ๔ELER, "La politique du père Hugo lu par la gauche juive-allemande (1840-1940)" \\
\hline $14 \mathrm{~h} 30$ & Sylvie APRILE, "Victor Hugo, écrivain engagé ou juge d'instruction de l'histoire ?" \\
\hline $15 \mathrm{~h}$ & Pause \\
\hline $15 \mathrm{~h} 15$ & $\begin{array}{l}\text { Jacqueline LALOUETTE, "La place et l'image de Victor Hugo dans les manuels d'histoire et } \\
\text { de lecture de l'école primaire" }\end{array}$ \\
\hline $15 \mathrm{~h} 45$ & François MARCOT, "Un siècle de célébration de Victor Hugo à Besançon" \\
\hline
\end{tabular}

Discussion

16h30 Conclusions par Annie STORA-LAMARRE

18h30 Conférence grand public, Petit Kursaal : Gaston BORDET "Victor Hugo penseur de la démocratie" 